\title{
Accumulating NH radicals in a magnetic trap
}

\author{
Sebastiaan Y. T. van de Meerakker, ${ }^{1}$ Rienk T. Jongma, ${ }^{1,2}$ Hendrick L. Bethlem, ${ }^{1,2}$ and Gerard Meijer ${ }^{1,2}$ \\ ${ }^{1}$ FOM-Institute for Plasmaphysics Rijnhuizen, P.O. Box 1207, NL-3430 BE Nieuwegein, The Netherlands \\ ${ }^{2}$ Department of Molecular and Laser Physics, University of Nijmegen, Toernooiveld 1, NL-6525 ED Nijmegen, The Netherlands
}

(Received 11 April 2001; published 13 September 2001)

\begin{abstract}
We propose a scheme to accumulate fermionic or bosonic $\mathrm{NH}$ radicals $\left({ }^{14} \mathrm{NH}\right.$ or ${ }^{15} \mathrm{NH}$, respectively) in a magnetic trap in order to increase phase-space density. $\mathrm{NH}$ molecules prepared in a long-lived metastable state that exhibits a large Stark effect can be brought to a standstill using Stark deceleration. By applying an intense laser field, the NH radicals can then be forced to spontaneously decay to the electronic ground state. In the ground state the NH molecules are no longer sensitive to electric fields but show a significant Zeeman effect instead, enabling confinement and reloading of ground-state molecules in a magnetic trap.
\end{abstract}

DOI: 10.1103/PhysRevA.64.041401

PACS number(s): 33.80.Ps, 33.55.Be, 33.70.Ca

The ability to cool and slow atoms with light for subsequent trapping allows investigation of the properties and interactions of the trapped atoms in unprecedented detail. This has led to exciting and sometimes unforeseen results, and the payoffs have included atom interferometry, precision spectroscopy, Bose-Einstein condensation, and even formation of atom lasers $[1,2]$. In principle, all these experiments could be performed with molecules as well [3]. Molecules would add an extra dimension to these experiments as they can be prepared in a wide variety of selected rotational and vibrational quantum states and as they can be spatially oriented. However, the complex structure of molecules has thus far prohibited the type of laser manipulation that has been so crucial for the success in the field of cold atoms. So far, three alternative techniques have been demonstrated for the trapping of molecules. In the first technique alkali-metal dimers, as can be formed through the association of ultracold alkali-metal atoms [4-6], are trapped in the focus of an intense laser beam [7]. In the second technique paramagnetic molecules in their lowest rotational levels are trapped in an inhomogeneous magnetic field after thermalization via collisions with a cold $\mathrm{He}(350 \mathrm{mK})$ buffer gas [8]. The third trapping technique, developed in our laboratory, exploits the interaction of neutral dipolar molecules with time-varying inhomogeneous electric fields. It resulted in confinement of ground-state ammonia molecules in an electrostatic trap [9] and in a storage ring [10], at temperatures that can be close to the temperature of the injected sample (down to a few mK [11]).

To be able to study molecular interactions and collective effects in these trapped cold molecular gases, with the experimental realization of molecular Bose-Einstein condensation and coherent molecular beams as the ultimate goal, the phase-space density needs to be further increased, i.e., the number density needs to be made higher and/or the temperature needs to be reduced. The Liouville theorem dictates that an increase of phase-space density is not possible without dissipation. Consequently, nonconservative forces are required to increase phase-space density. Evaporative cooling [12], a method that has been very successful for cold atoms, might be applicable to molecules as well once the number density in the trap is large enough that collisions occur. Although not experimentally realized yet, other cooling schemes like laser-cooling on rovibrational transitions or cavity-mediated cooling [13], have been suggested. For our technique, the most straightforward method to increase the phase-space density of trapped molecules would be the accumulation of several packages of molecules in the trap; the density that we have reached so far $\left(10^{6} / \mathrm{cm}^{3}\right)$ is obtained employing a single loading cycle [9]. Simply reloading the trap, however, requires opening up the trapping potential thereby losing the molecules that are already stored.

In this Rapid Communication we propose a scheme to reload a magnetic trap and to circumvent this fundamental obstacle, a scheme that works specifically for the $\mathrm{NH}$ radical. This scheme can be realized by producing NH molecules in the $a^{1} \Delta$ state, which can be slowed down to a complete standstill using our so-called "Stark decelerator" [11,14]. Subsequently, the molecules are forced to decay to the $X^{3} \Sigma^{-}$electronic ground state by exciting the spin-forbidden $A^{3} \Pi \leftarrow a^{1} \Delta$ transition. In the ground state the molecules are rather insensitive to electric fields, but experience a significant Zeeman shift allowing confinement of the ground state molecules in a magnetic trap. The "laser-driven" spontaneous decay process effectively switches off the electric-field interaction of the molecules enabling the accumulation of several packages of molecules in the magnetic trap, and automatically fulfills the requirement of a unidirectional pathway to the ground state.

In the scheme proposed here, ${ }^{14} \mathrm{NH}$ is used throughout, although the arguments hold equally well for ${ }^{15} \mathrm{NH}$. The electronic states of $\mathrm{NH}$ that are of relevance to the proposed scheme are schematically shown in the left part of Fig. 1. The Stark shift of the $a^{1} \Delta(v=0, J=2)$ state, located some $1.5 \mathrm{eV}$ above the electronic ground state, is plotted as a function of the electric-field strength in the upper inset to Fig. 1. The $\Lambda$-doublet splitting of this level is only $115 \mathrm{kHz}$ [15], and a nearly linear Stark shift is observed. The $a^{1} \Delta(v$ $=0, J=2, M \Omega=-4$ ) level, originating from the upper $\Lambda$-doublet component of $f$ parity, experiences a positive Stark shift of more than $3 \mathrm{~cm}^{-1}$ in an electric field of 200 $\mathrm{kV} / \mathrm{cm}$. Therefore, molecules in this state are excellent candidates to be slowed down with the Stark decelerator. It should be noted that the radiative lifetime of the $a^{1} \Delta$ state is very long (12.5 s [16]) compared to the typical duration of the deceleration process.

A setup that can be used to test the proposed scheme is 


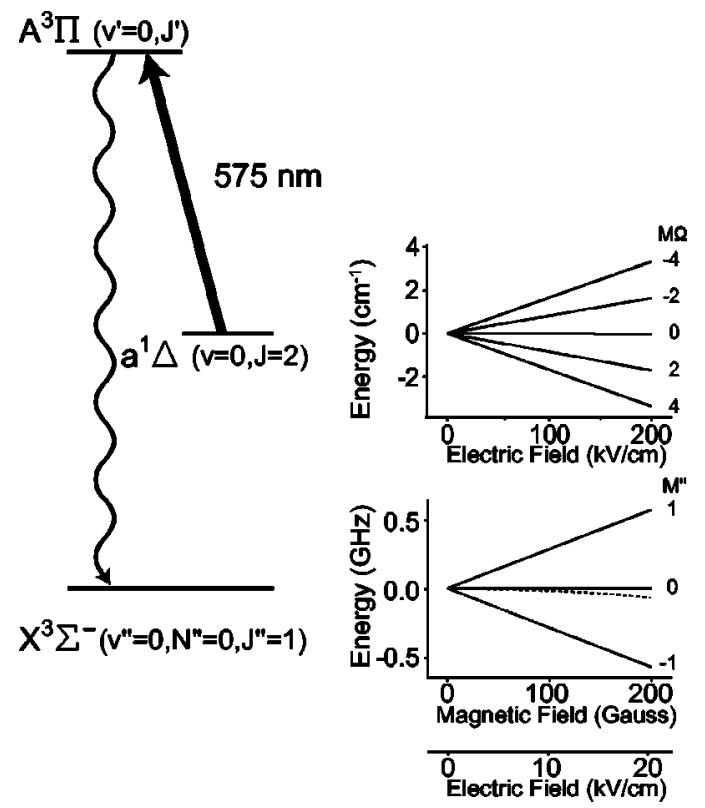

FIG. 1. The electronic states of $\mathrm{NH}$ that are of relevance to the proposed scheme. The $\mathrm{NH}$ molecules prepared in the long-lived $a^{1} \Delta(v=0, J=2)$ state exhibit a large Stark shift, as shown in the inset. By inducing the spin-forbidden $A^{3} \Pi\left(v^{\prime}=0\right) \leftarrow a^{1} \Delta(v=0)$ transition around $575 \mathrm{~nm}$, the molecules are forced to spontaneously decay to the $X^{3} \Sigma^{-}\left(v^{\prime \prime}=0\right)$ state. Both the Stark shift (dashed curve) and Zeeman shift (solid curves) of the lowest rotational level in this state are shown. For the Stark shift, the different $M$ components are unresolved. Note the different energy scale for both insets.

schematically depicted in Fig. 2. NH molecules in their metastable $a^{1} \Delta$ state are produced in a pulsed supersonic gas expansion from a precursor molecule. Making use of the process of adiabatic cooling, the rotational ground state ( $J$ $=2$ ) will be predominantly populated in the beam, and high densities of molecules in this state at a translational temperature of typically $1 \mathrm{~K}$ can be reached. Photodissociation of $\mathrm{HN}_{3}$ in the UV is known to yield NH in the $a^{1} \Delta$ state with an efficiency close to unity [17]. When applied in the expansion region of a pulsed supersonic beam, it has provided

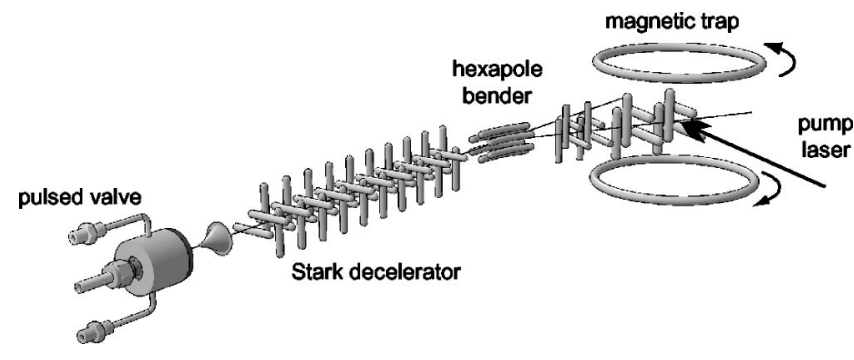

FIG. 2. A typical setup that can be used to accumulate $\mathrm{NH}$ molecules in a magnetic trap. NH molecules in the $a^{1} \Delta$ state are produced in a pulsed molecular beam from a precursor, and slowed down with the Stark decelerator. A hexapole bender injects only the selected bunch of molecules into the final part of the decelerator, where the molecules are stopped in the last, enlarged electric-field section. A pump laser provides the molecules a unidirectional path to the electronic ground state, where they are accumulated in a magnetic trap. rotationally cold $\mathrm{NH}$ molecules in the $a^{1} \Delta(v=0)$ state when $\mathrm{N}_{2}$ is used as a carrier gas [18]. In order to reduce the initial velocity of the molecular beam, either argon, krypton, or xenon can be coexpanded from the pulsed valve as a heavy inert carrier gas. Alternatively, $\mathrm{NH}\left(a^{1} \Delta\right)$ can be produced in an electrical discharge, by photolysis of $\mathrm{NH}_{3}$ [19] and HNCO [20], or from ground state $\mathrm{NH}$ by inducing the forbidden $a^{1} \Delta \leftarrow X^{3} \Sigma^{-}$transition around $790 \mathrm{~nm}$ [16].

The absolute velocity of the NH molecules needs to be largely reduced before the molecules can be trapped. This can be realized by passing the molecular beam through the Stark decelerator, thus producing high densities of slow, velocity-selected molecules [11,14]. The NH molecules can be brought to a complete standstill in the last, enlarged electric-field section of the decelerator in which the electric field will only go up to about $20 \mathrm{kV} / \mathrm{cm}$. A conventional magnetic trap, for instance, formed by two coils in the antiHelmholtz configuration, creates a magnetic-field minimum exactly at the position where the molecules are stopped. The Zeeman effect in the $a^{1} \Delta$ state due to the presence of the magnetic field is negligibly small compared to the Stark effect. As soon as the molecules in the $a^{1} \Delta$ state are brought to a standstill, the electric fields of the decelerator are switched off, while the magnetic field is permanently on. Immediately after switching off the electric field, the decelerated molecules can be pumped via a laser induced spinforbidden transition from the $a^{1} \Delta$ state to the lowest vibrational level of the $A^{3} \Pi$ state. Although this transition has, to the best of our knowledge, never been experimentally observed, we conclude from the analysis given below that it can be efficiently induced using high-intensity narrow-band radiation.

The lifetime of the electronically excited $A^{3} \Pi\left(v^{\prime}=0\right)$ state is around $450 \mathrm{~ns}$ [21], and a transition from this state to the $X^{3} \Sigma^{-}\left(v^{\prime \prime}=0\right)$ ground state will occur via spontaneous emission of a photon. The allowed $A^{3} \Pi \rightarrow X^{3} \Sigma^{-}$transition is almost perfectly "vertical" (the Franck-Condon factor for this vibrational transition is better than 0.999 [22]), and consequently molecules will end up in the lowest vibrational level of the electronic ground state with almost unit efficiency. Selected $A^{3} \Pi\left(v^{\prime}=0, J^{\prime}\right) \leftarrow a^{1} \Delta(v=0, J)$ transitions will cause a major fraction (up to $62 \%$, vide infra) of the excited molecules to end up in the $X^{3} \Sigma^{-}\left(v^{\prime \prime}=0, N^{\prime \prime}=0, J^{\prime \prime}\right.$ $=1, M^{\prime \prime}=1$ ) level.

Both the Zeeman shift (solid curves) and the Stark shift (dashed curve) of this rotational state are indicated in the lower inset to Fig. 1. The hyperfine structure is not included in the figure, as calculations show that it does not significantly influence the overall picture. It is observed that even in electric fields as high as $20 \mathrm{kV} / \mathrm{cm}$ the Stark shift is very small, making ground-state $\mathrm{NH}$ molecules rather insensitive to electric-fields. Due to the nonzero electron spin $(S=1)$, there is a significant Zeeman shift, allowing the confinement of ground state $\mathrm{NH}$ molecules in a magnetic trap. Using conventional coils, magnetic fields up to $100 \mathrm{G}$ are readily achieved, creating a $300 \mathrm{MHz}$, or $15 \mathrm{mK}$, deep trap that can store molecules in states that experience a positive Zeeman shift. The trap depth is nicely matched to the typical transla- 
tional temperature of the decelerated package of molecules exiting the Stark decelerator, which can be as low as a few $\mathrm{mK}[11]$.

The absence of a significant Stark shift in the electronic ground state allows the switching of the Stark decelerator to stop the next pulse of NH molecules in the $a^{1} \Delta$ state without losing the magnetically trapped ground-state molecules. The spontaneous emission step makes that the transfer of molecules from the $a^{1} \Delta$ to the $X^{3} \Sigma^{-}$state is unidirectional. Via this mechanism, Liouville's theorem is circumvented and an increase of phase-space density is obtained with every pulse of molecules that is stimulated to the $X^{3} \Sigma^{-}$state.

Obviously, a critical step in the proposed scheme is the realization of the spin-forbidden $A^{3} \Pi\left(v^{\prime}=0\right) \leftarrow a{ }^{1} \Delta(v$ $=0)$ transition, of which we here estimate the intensity. The $A^{3} \Pi$ state is best described intermediate between Hund's cases (a) and (b), and consists of three $\Omega$ manifolds exhibiting a rather large degree of mixing already for low values of $J$. The intensity of the forbidden transition arises from the fact that the spin-orbit interaction mixes some $c^{1} \Pi$ character into the $A^{3} \Pi_{1}$ wave function. Additionally, the $a^{1} \Delta$ and $b^{1} \Sigma^{+}$states couple to the $A^{3} \Pi_{2}$ and $A^{3} \Pi_{0}$ states, respectively. Since the spin-orbit coupling between the $c^{1} \Pi$ and $A^{3} \Pi_{1}$ states of NH is well documented [23], a realistic estimate of the lower limit for the transition dipole moment can be obtained by exclusively considering the ${ }^{1} \Pi-{ }^{3} \Pi$ coupling.

The description of this coupling for the $A^{3} \Pi \leftarrow a^{1} \Delta$ transition in $\mathrm{NH}$ is similar to the description of the $a^{3} \Pi$ $\leftarrow X^{1} \Sigma^{+}$transition in CO, for which James [24] calculated the ${ }^{1} \Pi-{ }^{3} \Pi$ mixing to experimental accuracy. In analogy to this analysis, the transition moment $\mu_{0,0}$ of the $A^{3} \Pi\left(v^{\prime}\right.$ $=0) \leftarrow a^{1} \Delta(v=0)$ transition in $\mathrm{NH}$ is given by

$$
\begin{aligned}
\mu_{0,0}= & \sum_{v^{\prime \prime \prime}} \frac{\left\langle A^{3} \Pi, v^{\prime}=0|\mathcal{H}| c^{1} \Pi, v^{\prime \prime \prime}\right\rangle}{E\left(A^{3} \Pi, v^{\prime}=0\right)-E\left(c^{1} \Pi, v^{\prime \prime \prime}\right)} \\
& \times\left\langle c^{1} \Pi, v^{\prime \prime \prime}|\mu(r)| a^{1} \Delta, v=0\right\rangle,
\end{aligned}
$$

where $\mathcal{H}$ represents the spin-orbit interaction, and $\mu(r)$ the transition dipole moment function for the $c^{1} \Pi \leftarrow a^{1} \Delta$ electric dipole allowed transition. The sum extends over all vibrational levels $v^{\prime \prime \prime}$ in the $c^{1} \Pi$ state, but is dominated by the contribution from the $v^{\prime \prime \prime}=0$ level. Using the potential energy functions and $\mu(r)$ from literature $[23,25]$, we obtain $\mu_{0,0} \geqslant 4.4 \times 10^{-4}$ atomic units (a.u.). The rotational transition probability to a $\left(J^{\prime}, M^{\prime}, e / f\right)$ level in one of the $A^{3} \Pi_{\Omega^{\prime}}$ manifolds is calculated by multiplying $\mu_{0,0}$ by the amount of $\Omega^{\prime}=1$ character in the particular manifold and by the appropriate Hönl-London factor for the $c^{1} \Pi\left(J^{\prime}, M^{\prime}, e / f\right)$ $\leftarrow a^{1} \Delta(J=2, M=2, f)$ transition.

Due to the Zeeman effect in both the $a^{1} \Delta$ and $A^{3} \Pi$ states, the $M$-resolved transition probability needs to be taken into account. In particular, a transition to either the $\left(J^{\prime}\right.$ $\left.=2, M^{\prime}=2, e\right)$ level in the $A^{3} \Pi_{2}$ manifold or the $\left(J^{\prime}\right.$ $\left.=1, M^{\prime}=1, f\right)$ level in the $A^{3} \Pi_{1}$ manifold is of interest. After the spontaneous decay process, $62 \%$ (when the $A^{3} \Pi_{2}$ state is excited) or $32 \%\left(A^{3} \Pi_{1}\right.$ state $)$ of the excited mol- ecules reside in the $X^{3} \Sigma^{-}\left(v^{\prime \prime}=0, N^{\prime \prime}=0, J^{\prime \prime}=1, M^{\prime \prime}=1\right)$ rotational ground state. We calculated the transition moments for these transitions as $\mu \geqslant 0.16 \times 10^{-4}$ a.u. and $\mu \geqslant 2.0$ $\times 10^{-4}$ a.u., respectively. It should be noted that by including the ${ }^{1} \Delta-{ }^{3} \Pi_{2}$ coupling, the value of $\mu$ for the transition to the $A^{3} \Pi_{2}\left(J^{\prime}=2, M^{\prime}=2, e\right)$ level might be significantly increased. In the discussion presented here, the hyperfine structure is neglected, and the transfer efficiencies are therefore the sum over all contributing hyperfine sublevels.

The strength of the two NH transitions can be directly compared to the strength of the $Q_{2}(1)$ line of the aforementioned transition in $\mathrm{CO}$, which equals $\mu=5.2 \times 10^{-4}$ a.u. The latter transition can be readily saturated with the spectrally bright laser radiation that can be obtained from a Pulsed Dye Amplifier system [11,14], providing typically 1 $\mathrm{mJ}$ of radiation around $206 \mathrm{~nm}$. For $\mathrm{NH}$, the $A^{3} \Pi\left(v^{\prime}=0\right)$ $\leftarrow a^{1} \Delta(v=0)$ transition is around $575 \mathrm{~nm}$, where narrowband $(\approx 100 \mathrm{MHz})$ radiation up to $100 \mathrm{~mJ}$ per pulse can be generated. Therefore, we are confident that both transitions can be induced under saturated conditions.

Considering the relatively high power that is needed for the pump laser, it is remarked that no (dissociative) resonances are expected at the two-photon level, both for molecules residing in the $a^{1} \Delta(v=0)$ state [25] and for groundstate molecules being already confined inside the trap. Also, problems related to rescattering of spontaneously emitted photons, observed in optically dense atomic clouds and providing a mechanism for loss and heating, are not likely to occur in dense clouds of $\mathrm{NH}$, as the electronic transition strength is much weaker than transitions typically used to cool and trap alkali-metal atoms.

Referring back to Fig. 2, collisions of molecules in the magnetic trap with the fast part of the next gas pulse that is not sufficiently decelerated, can be prevented by placing the last few stages of the decelerator under a small angle relative to the molecular beam axis. By using a pulsed hexapole bender [10] as indicated in Fig. 2, only the decelerated bunch of molecules, which is suitable to be trapped, will be coupled into the last few stages, while the remainder of the gas pulse passes in a straight line. The presence of $\mathrm{NH}$ molecules in the trap in either the $a^{1} \Delta$ or the $X^{3} \Sigma^{-}$state can be conveniently monitored via laser induced fluorescence detection on strong electronic transitions in the near-UV region of the spectra [26,27]. Considering the (almost) unity FranckCondon factor for the $A^{3} \Pi\left(v^{\prime}=0\right) \leftarrow X^{3} \Sigma^{-}\left(v^{\prime \prime}=0\right)$ transition [22], it is emphasized that an (almost) closed rovibrational transition can be found allowing further reduction of the temperature of $\mathrm{NH}$ radicals using conventional laser cooling after application of the Stark deceleration technique, although additional microwave transitions between hyperfine components will be needed to effectively close the absorption-emission cycle.

Compared to a single pulse, the gain in intensity of trapped molecules via accumulating molecules from consecutive cycles equals the product of the trapping time and the repetition rate at which the experiment is operated. Trapping times exceeding $10 \mathrm{~s}$ are anticipated at ultrahigh vacuum $\left(<10^{-10}\right.$ Torr $)$ conditions. The setup we are cur- 
rently using has a maximum repetition rate of $10 \mathrm{~Hz}$, yielding a gain in intensity of at least two orders of magnitude via the proposed accumulation process. The short duration of the deceleration process (on the order of $1 \mathrm{~ms}$ ) implies that the whole experiment can in principle be operated at higher repetition rates, increasing the gain correspondingly.
This work has been part of the research program of the "Stichting voor Fundamenteel Onderzoek der Materie (FOM)," which is financially supported by the "Nederlandse Organisatie voor Wetenschappelijk Onderzoek (NWO)." The research of R.T.J. has been made possible by the Royal Netherlands Academy of Arts and Sciences.
[1] S. Chu, Rev. Mod. Phys. 70, 685 (1998); C.N. CohenTannoudji, ibid. 70, 707 (1998); W.D. Phillips, ibid. 70, 721 (1998).

[2] M.H. Anderson et al., Science 269, 198 (1995).

[3] B. Levi, Phys. Today 53, 46 (2000).

[4] A. Fioretti et al., Phys. Rev. Lett. 80, 4402 (1998).

[5] A.N. Nikolov et al., Phys. Rev. Lett. 84, 246 (2000).

[6] C. Gabbanini et al., Phys. Rev. Lett. 84, 2814 (2000).

[7] T. Takekoshi, B.M. Patterson, and R.J. Knize, Phys. Rev. Lett. 81, 5105 (1998).

[8] J.D. Weinstein et al., Nature (London) 395, 148 (1998).

[9] H.L. Bethlem et al., Nature (London) 406, 491 (2000).

[10] F.M.H. Crompvoets et al., Nature (London) 411, 174 (2001).

[11] H.L. Bethlem et al., Phys. Rev. Lett. 84, 5744 (2000).

[12] W. Ketterle and N.J. van Druten, Adv. At., Mol., Opt. Phys. 37, 181 (2000).

[13] V. Vuletić and S. Chu, Phys. Rev. Lett. 84, 3787 (2000).

[14] H.L. Bethlem, G. Berden, and G. Meijer, Phys. Rev. Lett. 83, 1558 (1999).
[15] W. Ubachs et al., J. Mol. Spectrosc. 115, 88 (1986).

[16] J.L. Rinnenthal and K.H. Gericke, J. Mol. Spectrosc. 198, 115 (1999).

[17] J.R. McDonald, R.G. Miller, and A.P. Baronavski, Chem. Phys. Lett. 51, 57 (1977).

[18] D.G. Sauder, D. Patel-Misra, and P.J. Dagdigian, J. Chem. Phys. 91, 5316 (1989).

[19] R.D. Kenner, F. Rohrer, and F. Stuhl, J. Chem. Phys. 86, 2036 (1987).

[20] W.S. Drozdoski, A.P. Baronavski, and J.R. McDonald, Chem. Phys. Lett. 64, 421 (1979).

[21] R.D. Kenner et al., J. Chem. Phys. 91, 1440 (1989).

[22] D.R. Yarkony, J. Chem. Phys. 91, 4745 (1989).

[23] B. Bohn et al., J. Chem. Phys. 96, 5059 (1992).

[24] T.C. James, J. Chem. Phys. 55, 4118 (1971).

[25] D. Patel-Misra et al., J. Chem. Phys. 94, 1913 (1990).

[26] C.R. Brazier, R.S. Ram, and P.F. Bernath, J. Mol. Spectrosc. 120, 381 (1986).

[27] R.S. Ram and P.F. Bernath, J. Opt. Soc. Am. B 3, 1170 (1986). 